\title{
INITIAL IMAGING OF 7-GEV ELECTRON BEAMS WITH OTR/ODR TECHNIQUES AT THE APS*
}

\author{
A.H. Lumpkin ${ }^{\#}$, W.J. Berg, N.S. Sereno, and C.Y. Yao \\ Advanced Photon Source, Argonne National Laboratory \\ Argonne, Illinois 60439 U.S.A.
}

\begin{abstract}
The development of nonintercepting (NI) diagnostics for beam size continues to be of interest in the accelerator community. In the three rings of the Advanced Photon Source (APS) facility, we use optical and x-ray synchrotron radiation generated as the electron beam transits the dipole magnetic fields as an NI mechanism to image the beam during top-up operations. However, in the straight transport lines an alternate beam-size measurement method is needed. Optical diffraction radiation (ODR) is under investigation to monitor $7-\mathrm{GeV}$ beam size and trajectory in the booster synchrotron-tostorage ring (BTS) beamline during top-up operations. We have performed our initial measurements with an Aluminum blade/mirror that served as an optical transition radiation (OTR) monitor when fully inserted into the beam and as an ODR monitor when the beam passed near the edge.
\end{abstract}

\section{INTRODUCTION}

The development of nonintercepting (NI) diagnostics continues to be of interest at the Advanced Photon Source (APS) as well as elsewhere. In the two injector rings and the storage ring we use optical and $\mathrm{x}$-ray synchrotron radiation (OSR and XSR, respectively) generated as the electron beam transits the dipole magnetic fields as an NI mechanism to image the beam during top-up operation. However, in the straight transport lines an alternate method to measure beam size is needed. Optical diffraction radiation (ODR) is under investigation [1-6] to monitor beam size and beam trajectory in the booster synchrotron-to-storage ring (BTS) beamline during top-up operations. We have performed our initial measurements with an Al blade/mirror that served as an optical transition radiation (OTR) monitor when fully inserted into the beam and as an ODR monitor when the beam passed near the edge. We have tracked the signal generated by the $\mathrm{Al}$ metal surface as the beam passed nearby as a function of the impact parameter $d$ (beam center distance from the mirror edge). Because the beam has an elliptical shape $\sigma_{\mathrm{x}} / \sigma_{\mathrm{y}}=7$ as measured by OTR, we have performed the experiments on horizontal beam size with a vertical plane mirror. Due to the large Lorentz factor, $\gamma \approx 14,000$ for the $7-\mathrm{GeV}$ beam, and the fact that appreciable ODR is emitted from the metal when $\gamma \lambda \approx d$, we see visible ODR radiation for impact parameters of 1 to $2 \mathrm{~mm}$. This

\footnotetext{
*Work supported by the U.S. Department of Energy, Office of Basic Energy Sciences, under Contract No. W-31-109-ENG-38.

"lumpkin@aps.anl.gov
}

allowed us to initiate our experiments with standard optics and imaging techniques. Further details will be presented in the next sections.

\section{EXPERIMENTAL AND ODR BACKGROUND}

A layout of the nonintercepting beam diagnostics throughout the APS facility is shown in Fig. 1 of ref. [7] as described previously. Within the linac, a chicane bunch compressor provides the dipole magnets that might be used to generate OSR for those beams. These imaging stations are not yet built. In the particle accumulator ring (PAR) and the booster synchrotron we have operational OSR ports for monitoring beam size, position, and bunch length. Our target in this paper is the booster synchrotron-to-storage ring (BTS) transport line, which currently only has intercepting screens as beam size and profile monitors. We have a test line also at the exit from the booster where we have installed the OTR/ODR station. Downstream of a dipole magnet that directs beam to this line we have an $\mathrm{rf}$ beam position monitor (BPM), the OTR/ODR station, a localized loss monitor that employs a Cherenkov radiation detector, a Chromox beam profile screen, and the beam dump. These are shown in the schematic in Fig. 1.

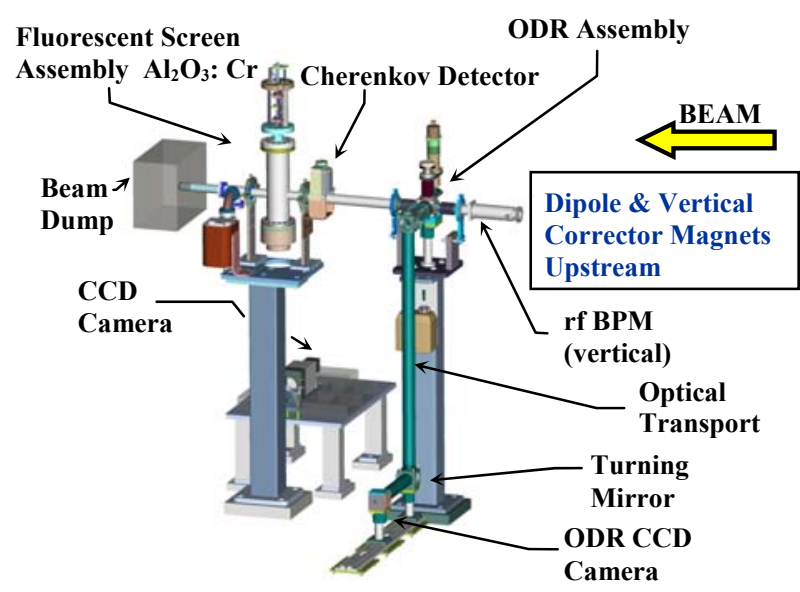

Figure 1: A schematic drawing of the rf BPM, ODR assembly, Cherenkov detector, beam profile screen, and beam dump. The optical transport to the CCD camera is also indicated.

The OTR/ODR converter is an $\mathrm{Al}$ blade/mirror that is $1.5 \mathrm{~mm}$ thick, $30 \mathrm{~mm}$ wide, and $30 \mathrm{~mm}$ tall. It is oriented with its surface normal at $45^{\circ}$ to the beam direction so that the OTR or ODR is directed at $90^{\circ}$ to the beam direction 
and into the optical transport. The optical transport consists of a turning mirror at $22 \mathrm{~cm}$ from the source, an achromatic triplet at $30 \mathrm{~cm}$ from the source, a singlet lens, another turning mirror, and an achromatic singlet at $120 \mathrm{~cm}$ from the source. A standard Sony CCD camera is used to image the signal. The optics magnification is such as to cover the width of the $30-\mathrm{mm}$ screen times $1 / \sqrt{2}$ and the vertical position of the blade edge $7.5 \mathrm{~mm}$ below the centerline. The ODR converter is mounted on a rod controlled by a stepper motor. The blade edge can be vertically positioned to $\pm 10 \mu \mathrm{m}$ over a 27.5 -mm span. There is a potentiometer-based readback. The CCD camera video signal is sent to a Data Cube MV200 digitizer for on-line processing and to allow capture of images for off-line analysis. The Cherenkov detector is located $0.6 \mathrm{~m}$ downstream of the blade-beam interaction point to measure localized beam-loss effects, or more precisely, to verify minimal beam loss on the blade during ODR measurements. The downstream profile screen is used in combination with setting the blade edge on centerline to set the beam trajectory. The upstream vertical correctors were also used to adjust the upstream steering so weak OSR signals were below beam centerline while the beam spot is bifurcated by the centered blade edge.

In the case of ODR, appreciable signal is emitted by the metal when $\gamma$ times $\lambda$, the reduced wavelength, is comparable to the impact parameters, where $\gamma$ is the Lorentz factor. Visible light optics and a standard CCD camera could thus be used. We attribute the near-field signal for $1.5-$ to $3.0-\mathrm{mm}$ impact parameters predominately to the ODR mechanism. A schematic of the process is shown in Fig. 2, except we have only the upper plane in our experiment.

\section{RESULTS AND DISCUSSION}

As a reference, we verified the $7-\mathrm{GeV}$ beam size via OTR with the blade edge inserted to $4.0 \mathrm{~mm}$ below beam centerline. The observed OTR image for a 0.4-nC pulse is shown in Fig. 3. The observed sizes are $\sigma_{x}=1375 \pm 25$ $\mu \mathrm{m}$ and $\sigma_{\mathrm{y}}=200 \pm 25 \mu \mathrm{m}$. This large ellipticity is why we chose to measure the horizontal size with a vertical blade first. Although an overlap point with 0.4-nC and 3.3-nC charge in the beam pulse was taken at $d=0.75$ $\mathrm{mm}$ (above beam centerline), we show the image with $d=$ $2.0 \mathrm{~mm}$ in Fig. 4 for a beam charge of $3.3 \mathrm{nC}$. The image intensity is reasonable (but $1 / 10$ of OTR) at $1.25 \mathrm{~mm}$. It is noticeably weaker and shorter in vertical extent for the $d=2.0 \mathrm{~mm}$ case. To validate this point we illuminated the screen, which allowed the beam image and the blade boundary to be seen. A more complete sampling of the signal versus blade position is given in Fig. 5. The signal peak position clearly moves in a linear correlation with the blade edge position as readback from the stepper motor assembly.

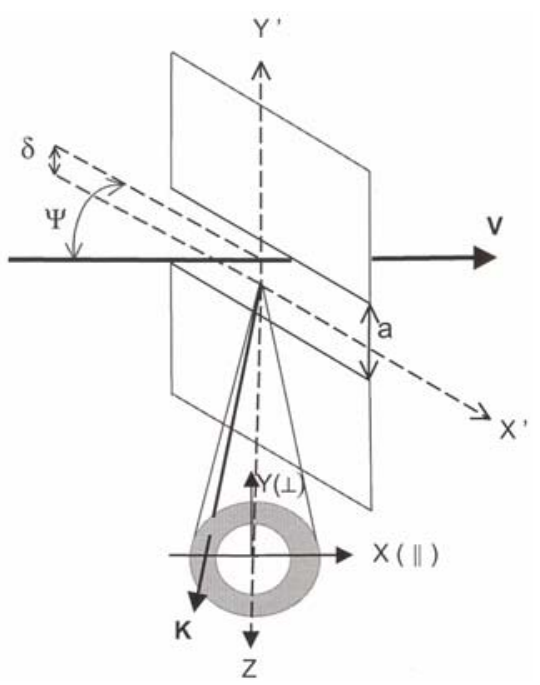

Figure 2: A schematic of the generation of ODR by a charged particle beam as it passes near a metal plane (based on Fig. 1 of reference 4.)

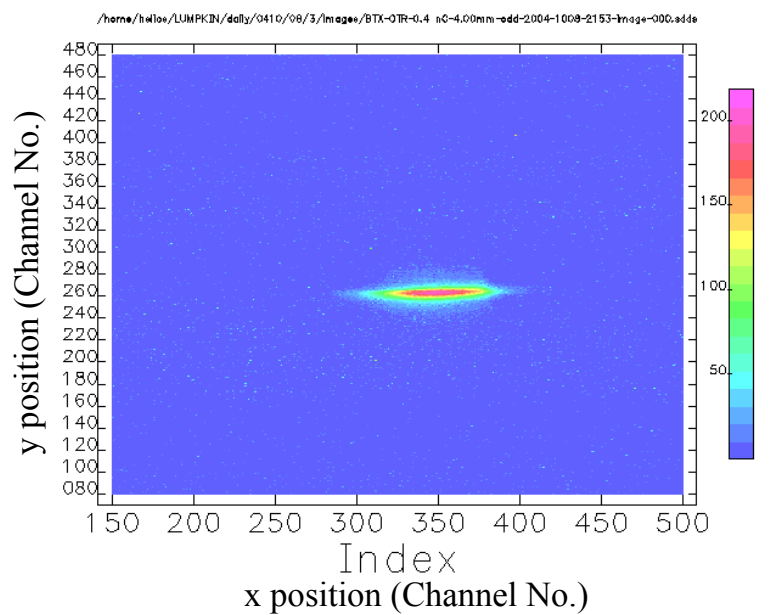

Figure 3: The observed OTR image of the $7-\mathrm{GeV}$ beam with $\mathrm{Q}=0.4 \mathrm{nC}$.

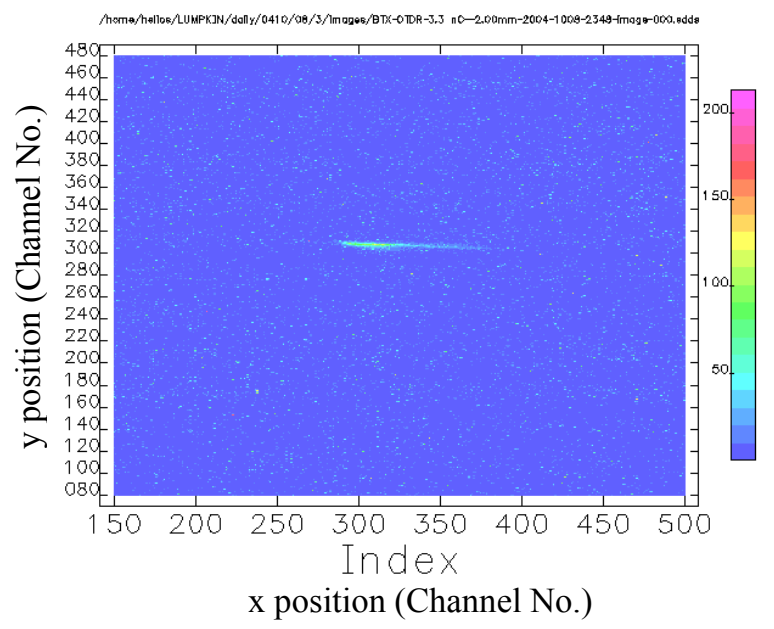

Figure 4: The observed ODR "image" of the 7-GeV beam with $\mathrm{Q}=3.3 \mathrm{nC}$ and $d=2.00 \mathrm{~mm}$. 


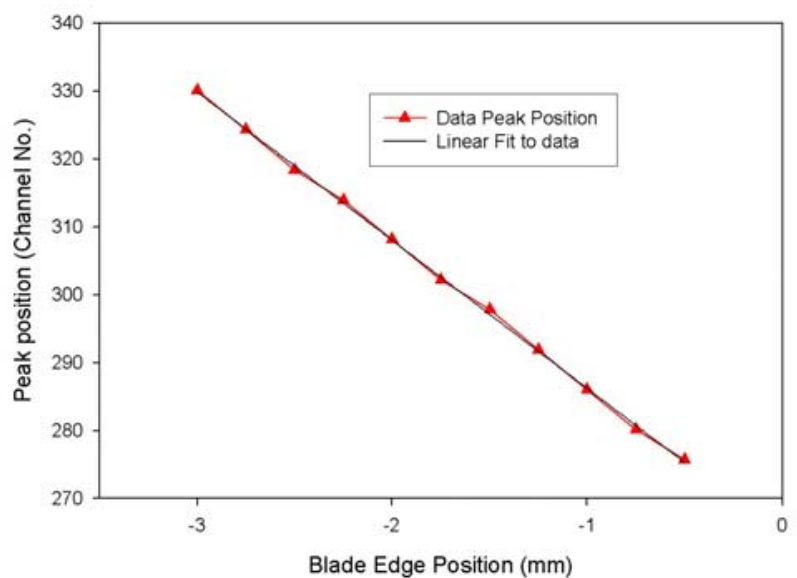

Figure 5: A plot of the linear dependence in the signal peak position versus the blade edge position.

The signal intensity is also tracked in Fig. 6 as a function of impact parameter. In this case an exponential decay is seen with argument $(-d / \gamma \lambda)$ and $\gamma \lambda=928 \mu \mathrm{m}$. This implies the effective observation wavelength was $0.420 \mu \mathrm{m}$, in the blue end of the visible spectrum.

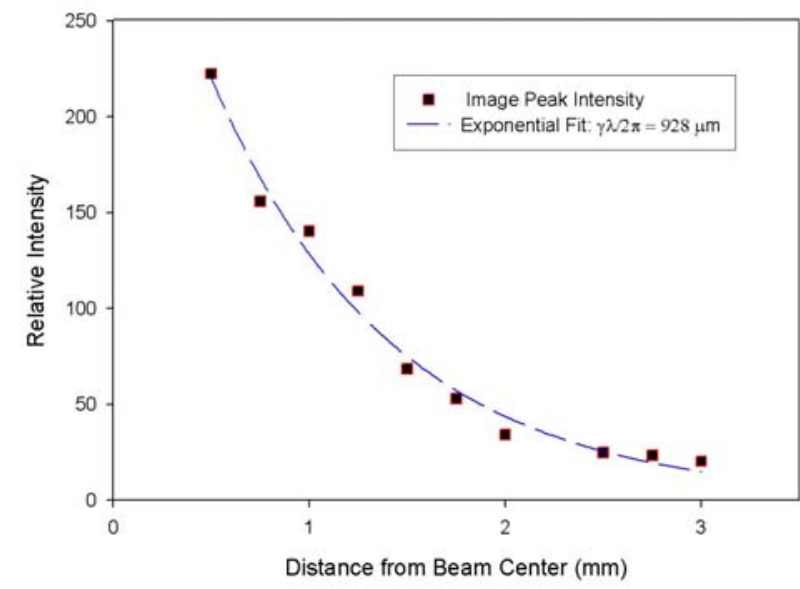

Figure 6: A plot of the experimental dependence of the signal peak intensity versus the blade edge position.

As an additional test, we measured the horizontal image position as a function of the upstream dipole power supply current for $d=0.75 \mathrm{~mm}$. The results are shown in Fig. 7. A slope of $0.508 \pm 0.006 \mathrm{~mm} / \mathrm{A}$ was determined. The individual points are 10-shot averages with the error bars calculated by the SDDS application program [8]. The experiment was done via a script that changed the dipole power supply set point and then tracked the process variables from the upstream current monitor, the rf BPM, the Cherenkov detector, and the video digitizer. The Cherenkov data are consistent with minimal beam losses at the blade, so the signal is predominately ODR. These data show sensitivity to $50-\mu \mathrm{m}$ centroid shifts for the $1375-\mu \mathrm{m}$-size beam. We expect that, with a smaller inherent beam size and higher optical magnification, ODR could be used to sense $10-\mu \mathrm{m}$ position changes or better.

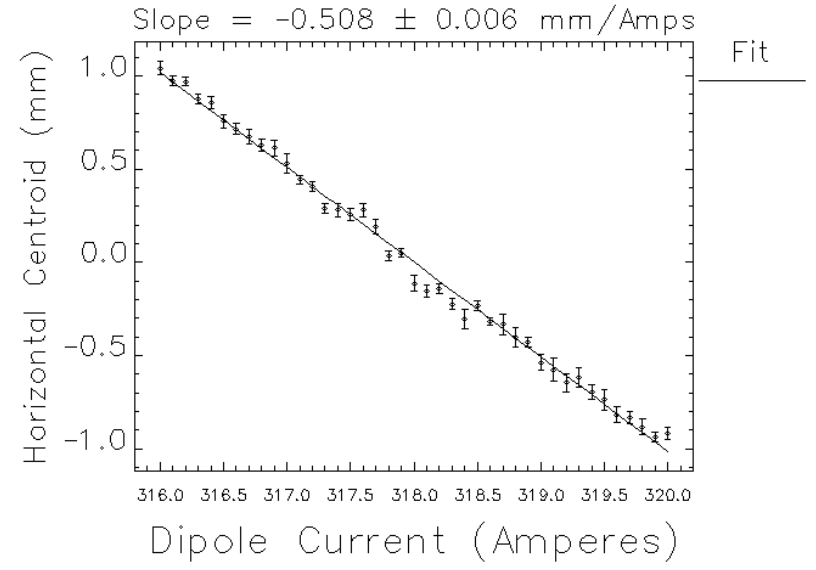

Figure 7: A plot of the ODR horizontal image centroid versus the dipole magnet current supply set point. A 50$\mu \mathrm{m}$ relative position sensitivity is observed.

\section{SUMMARY}

In summary, we have demonstrated for the first time an NI beam size measurement of a $7-\mathrm{GeV}$ beam using nearfield ODR imaging. We project that beam size monitoring should be possible with sub-50- $\mu \mathrm{m}$ resolution and position monitoring to the $10-\mu \mathrm{m}$ regime with a smaller beam. Although ODR is weaker than OTR, it has sufficient signal strength at $3.3 \mathrm{nC}$ of a single pulse for $d$ $\sim \gamma \lambda$. Obviously, in applications with higher average currents such as in $\mathrm{x}$-ray FELs, energy recovering linacs, and linear colliders, this signal strength will not be an issue. We have proposed experiments at SPPS at $28 \mathrm{GeV}$ in the coming year and hope to elucidate the phenomena.

\section{REFERENCES}

[1] M.L. Ter-Mikaelian, High Energy Electromagnetic Processes in Condensed Media (Wiley/Interscience, New York, 1972).

[2] D.W. Rule, R.B. Fiorito, and W.D. Kimura, AIP Conf. Proc. 390 (1997) 510.

[3] M. Castellano, Nucl. Instrum. Methods Phys. Res. A 394 (1997) 275.

[4] R.B. Fiorito and D.W. Rule, Nucl. Instrum. Methods Phys. Res. B 173 (2001) 67.

[5] P. Karataev et al., PRL 93 (2004) 244802.

[6] A.H. Lumpkin et al., "First Near-Field Imaging of Optical Diffraction Radiation Generated by a $7-\mathrm{GeV}$ Electron Beam,” submitted to Phys. Rev. Lett., May 2005.

[7] A.H. Lumpkin, W.J. Berg, and B.X. Yang, AIP Conf. Proc. 648 (2002) 433.

[8] H. Shang et al., Proc. of PAC 2003, p. 3470 (2003), http://www.jacow.org. 